\title{
Expression of $\alpha$ and $\beta$ subunits of the integrin superfamily in articular cartilage from macroscopically normal and osteoarthritic human femoral heads
} Keld Ostergaard, Donald M Salter, Jorgen Petersen, Klaus Bendtzen, Jesper Hvolris,
Claus B Andersen
Institute for

Inflammation

Research (IIR), RHIMA-Centre, National University Hospital/Rigshospitalet, Copenhagen, Denmark K Ostergaard

J Petersen

K Bendtzen

\section{Department of} Pathology, University of Edinburgh, Medical School, Edinburgh,

United Kingdom

D M Salter

Department of Orthopaedics, Hvidovre University Hospital, Hvidovre, Denmark

J Hvolris

Department of Pathology, National University Hospital/Rigshospitalet, Copenhagen, Denmark C B Andersen

Correspondence to: Dr K Ostergaard, Institute for Inflammation Research (IIR), 7541, RHIMA-Centre, National University Hospital/Rigshospitalet, Tagensvej 22, DK-2200 Copenhagen N, Denmark.

\begin{abstract}
Objective-The objective of this study was to detail the topographical and zonal distribution of $\alpha$ and $\beta$ subunits of the integrin superfamily in normal and osteoarthritic cartilage.
\end{abstract}

Methods-Immunohistochemistry utilising antibodies towards $\alpha$ and $\beta$ subunits was performed on cryostat sections of human articular cartilage from macroscopically normal $(n=6)$ and osteoarthritic $(n=6)$ femoral heads. Samples of articular cartilage were obtained from 12 topographically distinct sites from each femoral head. Each section was divided into zones (superficial, middle, deep) and staining scores were recorded.

Results-Normal cartilage stained for integrin subunits $\alpha 1, \alpha 5, \alpha V, \beta 1, \beta 4$, and $\beta 5$, but not for $\alpha 2, \alpha 3, \alpha 4, \alpha 6, \beta 2, \beta 3$, and $\beta 6$. Intact and non-intact residual cartilage from osteoarthritic femoral heads stained for $\alpha 1, \alpha 2, \alpha 5, \alpha V, \beta 1, \beta 4$, and $\beta 5$. Staining was occasionally seen for $\alpha 4$ and $\beta 2$, but not for $\alpha 3, \alpha 6, \beta 3$, and $\beta 6$. There was no topographical variation in the staining for any of the subunits in either normal or osteoarthritic cartilage. The only subunit that displayed a zonal variation was $\alpha \mathrm{V}$; staining for this subunit was most pronounced in the superficial zone compared with the middle and deep zones.

Conclusion-Chondrocytes in normal and osteoarthritic cartilage express the integrin subunits $\alpha 1, \alpha 5, \alpha \mathrm{V}, \beta 1, \beta 4$, and $\beta 5$. Chondrocytes in osteoarthritic cartilage, in addition, express the $\alpha 2, \alpha 4$, and $\beta 2$ subunits. The $\alpha v$ subunit is expressed by more chondrocytes in the superficial zone in comparison with cells in the deeper zones. None of the subunits display topographical variation in expression.

(Ann Rheum Dis 1998;57:303-308)

Interactions between chondrocytes and extracellular matrix in articular cartilage are critical to cell anchorage, matrix biosynthesis, and matrix degradation. ${ }^{12}$ Cell adhesion molecules are essential mediators of these interactions and may play an important part as environmental monitors involved in the regulation of cartilage matrix turnover. ${ }^{34}$ Human articular chondrocytes are known to express a range of cell adhesion molecules including cluster of differentiation (CD) 44 of the hyaluronan binding protein family, ${ }^{5}{ }^{6}$ intercellular adhesion molecule (ICAM) -1 of the immunoglobulin superfamily, ${ }^{78}$ and members of the integrin superfamily. ${ }^{9-11}$ In normal articular cartilage from femoral condyles, tibial plateaus, and taluses the integrin heterodimers $\alpha 1 \beta 1, \alpha 5 \beta 1$, and $\alpha \mathrm{V} \beta 5$ are expressed strongly and the heterodimers $\alpha 3 \beta 1$ and $\alpha \mathrm{V} \beta 3$ weakly. ${ }^{9-11}$ The subunits $\alpha 2$ and $\alpha 6$ have been demonstrated on fetal articular chondrocytes. ${ }^{10} 12$ Potential ligands of integrins expressed by articular cartilage chondrocytes such as collagens, fibronectin, vitronectin, thrombospondin, and laminin are prominent components of the pericellular matrix. ${ }^{1314}$

In normal human femoral head articular cartilage, thickness and composition vary with topography. ${ }^{15}{ }^{16}$ In addition, the morphology of chondrocytes, cellularity, and distribution of extracellular matrix components vary with depth. ${ }^{17-20}$ In osteoarthritic cartilage, qualitative alteration of the collagen content, ${ }^{21}{ }^{22}$ pericellular loss of collagen type II, ${ }^{23}$ neoexpression of collagen types I and III, ${ }^{24-26}$ increased expression of collagen type $\mathrm{VI},{ }^{27} \mathrm{IX}^{28}$ and $\mathrm{X}^{23}{ }^{29}$ and increased levels of fibronectin ${ }^{30} 31$ have been observed. The $\alpha \mathrm{V}$ subunit containing integrins have been immunolocalised preferentially to the superficial zone of normal articular cartilage. ${ }^{9}$ However, it has not been reported whether the expression of integrins also reflects the topographical heterogeneity and variation in composition of normal articular cartilage, and the expression of integrins in osteoarthritic cartilage has not been detailed.

The objective of this study was, therefore, to detail the topographical and zonal distribution of $\alpha$ and $\beta$ subunits of the integrin superfamily in normal and osteoarthritic cartilage from human femoral heads.

\section{Methods}

NORMAL CARTILAGE

Samples of macroscopically normal articular cartilage (Collins/McElligott grade 0$)^{32}$ were obtained at necropsy within 24 hours of death from two women (age 66 and 82 years) and four men (median age 73 years, range 45-88). None of the subjects had a clinical history of arthritis or chronic systemic inflammatory disease.

Samples for cryostat sections were collected from anterior, posterior, lateral, and medial aspects of perifoveal, central, and peripheral 
Table 1 Primary antibodies

\begin{tabular}{llll}
\hline Specificity & Clone number & Source & Dilution \\
\hline$\alpha 1$ & TS2/7 & $\begin{array}{l}\text { Dr F Sanchez-Madrid, Universidad Autónomale, } \\
\text { Madrid, Spain }\end{array}$ & $1: 15$ \\
$\alpha 2$ & P1E6 & $\begin{array}{l}\text { Life Technologies, Paisley, UK } \\
\text { Becton Dickenson, San Jose, CA, USA }\end{array}$ & $1: 1000$ \\
$\alpha 3$ & P1B5 & Biogenesis, Poole, UK & $1: 1000$ \\
$\alpha 4$ & P4G9 & Dr C Damsky, University of California, San Francisco, & $1: 100$ \\
$\alpha 5$ & BIIG2 & CA, USA & $1: 1000$ \\
$\alpha 6$ & GOH3 & Serotec, Kidlington, Oxford, UK & $1: 2000$ \\
$\alpha$ V & VNR147 & Chemicon International, Danvers, MA, USA & $1: 1000$ \\
$\beta 1$ & TS2/16 & Dr F Sanchez-Madrid, Universidad Autónomale, & \\
$\beta 2$ & MHM23 & Madrid, Spain & DAKO, Glostrup, Denmark \\
$\beta 3$ & Y2/51 & DAKO, Glostrup, Denmark & $1: 200$ \\
$\beta 4$ & 3E1 & Chemicon International, Danvers, MA, USA & $1: 100$ \\
$\alpha$ V35 & P1F6 & Life Technologies, Paisley, UK & $1: 1000$ \\
$\beta 6$ & E7P6G10P0 & Dr D Sheppard, University of California, San Francisco, & $1: 1000$ \\
& & CA, USA & \\
& & &
\end{tabular}

areas of femoral heads. A total of 12 triangular $(5 \times 5 \times 5 \mathrm{~mm})$ full thickness samples of articular cartilage were collected from each femoral head, snap frozen in liquid nitrogen, and stored in cryovials at $-80{ }^{\circ} \mathrm{C}$. The samples were later cut in $4 \mu \mathrm{m}$ thick cryostat sections and mounted on SuperFrost/Plus glass slides (Eire Scientific, Portsmouth, NH, USA) followed by fixation with acetone for 10 minutes.

OSTEOARTHRITIC CARTILAGE

Samples of femoral head articular cartilage were obtained from four women (median age 78 years, range $77-85$ ) and two men (age 68 and 71 years) undergoing replacement surgery for osteoarthritis of the hip. All femoral heads contained areas of denuded bone. The overall Collins/McElligott ${ }^{32}$ grades of osteoarthritis were III and IV. The samples included a spectrum of histological changes; intact cartilage with all zones preserved, non-intact cartilage showing fibrillation and cluster formation, and non-intact cartilage in which the superficial and middle zones were absent and only part of the deep zone remained. A total of up to 12 samples of residual articular cartilage were collected and processed as above.

All samples from both normal and osteoarthritic femoral heads were obtained from white subjects.

\section{IMMUNOHISTOCHEMISTRY}

Immunohistochemistry was carried out using a Shandon Sequenza (Life Science International, Basingstoke, UK) to achieve consistency of staining quality and to prevent sections from floating off.

The immunohistochemical staining procedure has been described previously. ${ }^{611}$ In brief: non-specific background staining was blocked by incubating the sections for 20 minutes with $100 \mu \mathrm{l}$ of normal rabbit serum (DAKO, Glostrup, Denmark) in TRIS buffered saline (TBS) (1:5). The primary antibody $(100 \mu \mathrm{l})$ was diluted in normal rabbit serum in TBS and allowed to incubate for 30 minutes at room temperature. After washing twice in $2 \mathrm{ml}$ of TBS for five minutes, $100 \mu$ l biotinylated rabbit antimouse immunoglobulins (DAKO) diluted $1: 400$ in normal rabbit serum in TBS was added for 30 minutes, followed by another wash. Antibody binding was visualised with an avidin and biotinylated horseradish peroxidase complex $(100 \mu \mathrm{l})(\mathrm{DAKO})$, which was diluted according to the DAKO protocol and incubated for 30 minutes, followed by washing and $100 \mu \mathrm{l}$ of 3-amino-9-ethylcarbazole (Sigma, St Louis, MO, USA) for 20 minutes. After another wash, the sections were counterstained with Mayer's haematoxylin, washed again, and mounted with coverslips.

Table 1 shows the primary antibodies used in this study.

\section{PROCEDURE CONTROLS}

Negative controls were provided by omitting the primary antibody and also by substituting non-immune mouse immunoglobulins (DAKO) for the primary antibody. No attempt was made to block for endogenous peroxidase as no endogenous peroxidase activity was seen in the negative controls. External positive tissue controls included sections of normal human tissue from skin and tonsils. Internal tissue controls were present in many of the sections; integrin positive cells include osteocytes ( $\alpha 4$, $\alpha 5, \alpha \mathrm{V}, \beta 1)$, osteoblasts $(\alpha 4, \alpha 5, \alpha \mathrm{V}, \beta 1)$, osteoclasts $(\alpha 2, \alpha 5, \alpha \mathrm{V}, \beta 1, \beta 3)$, fibroblasts $(\alpha 2, \alpha 5$, $\beta 1)$, and endothelial cells $(\alpha 1, \alpha 2, \alpha 3, \alpha 4, \alpha \mathrm{V}$, $\beta 1, \beta 2, \beta 3, \beta 4, \beta 5){ }^{13} 33$

To assess if the accessibility of integrin subunit epitopes to antibodies was hindered by extracellular matrix interactions, sections were treated with hyaluronidase $(10 \mathrm{mg} / \mathrm{ml})$ (Sigma), collagenase $(10 \mathrm{mg} / \mathrm{ml})$ (Sigma), or a combination thereof (hyaluronidase $2 \mathrm{mg} / \mathrm{ml}$; collagenase $10 \mathrm{mg} / \mathrm{ml}$ ) for 5-30 minutes at $37^{\circ} \mathrm{C}$ before the standard immunohistochemical staining.

\section{MICROSCOPIC EVALUATION}

As the samples for cryostat sections were all triangularly shaped in their horizontal dimension, a section of intact cartilage sampled and cut correctly would be rectangular in shape in the vertical dimension. Only sections of intact cartilage that clearly displayed the correct vertical orientation and thus contained an articular surface and calcified cartilage with a tidemark were considered for further examination. Also considered for examination were sections of non-intact residual cartilage from osteoarthritic joints that contained calcified cartilage with a tidemark. Only the deep zone or whatever part of the deep zone present (that is, the zone adjacent to the tidemark) was considered for examination in these sections.

The full section of intact cartilage from articular surface to tidemark was divided into quarters, as described previously. ${ }^{6}$ The quarter of the full section of cartilage that includes the surface was named the superficial zone. The half of the full section of cartilage below the superficial zone was named the middle zone. The quarter of the full section of cartilage below the middle zone and above the tidemark was named the deep zone. The calcified zone of articular cartilage was not included in the evaluation. Occasionally, the superficial zone was covered by a fibrous pannus, which was not included in the zonal division of the section.

Integrin staining for each zone was scored as: $4=$ all chondrocytes positive $3=$ more positive 
Table 2 Integrin subunits in articular cartilage from macroscopically normal femoral heads

\begin{tabular}{ll}
\hline $\begin{array}{l}\text { Integrin subunit } \\
\text { sections stained) }\end{array}$ & Median score (range) \\
\hline$\alpha 1(59)$ & $3(0-3)$ \\
$\alpha 2(71)$ & $0(-)$ \\
$\alpha 3(66)$ & $0(-)$ \\
$\alpha 4(71)$ & $0(-)$ \\
$\alpha 5(52)$ & $2(0-4)$ \\
$\alpha 6(72)$ & $0(-)$ \\
$\alpha \mathrm{V}(58)$ & $3(1-4)$ superficial \\
& $2.5(1-3)$ middle \\
$\beta 1(48)$ & $2(0-3)$ deep \\
$\beta 2(60)$ & $2(0-3)$ \\
$\beta 3(66)$ & $0(-)$ \\
$\beta 4(53)$ & $0(-)$ \\
$\alpha \mathrm{V} \beta 5(65)$ & $0(0-1)$ \\
$\beta 6(72)$ & $2(1-3)$ \\
& $0(-)$
\end{tabular}

All topographical sites and zones of 4-6 subjects were scored together except for $\alpha \mathrm{V}$ subunit where the score is given for the together except for $\alpha V$ subunit where the score is given for the
superficial, middle, and deep zone individually. Scores: $0=$ no positive chondrocytes; $1=$ more negative than positive chondrocytes; $2=$ positive and negative chondrocytes of approximately equal number; $3=$ more positive than negative chondrocytes; 4 all chondrocytes positive.

Table 3 Zonal distribution of aV subunit in articular cartilage from macroscopically normal femoral heads

\begin{tabular}{llllll}
\hline Score & 0 & 1 & 2 & 3 & 4 \\
\hline Superficial zone & 0 & 5 & 11 & 39 & 3 \\
Middle zone & 0 & 6 & 20 & 32 & 0 \\
Deep zone & 2 & 13 & 19 & 24 & 0
\end{tabular}

Numbers for the three zones represent sections ( 58 in total from six subjects) which have received a score. Scores: see table 2 .

than negative chondrocytes; $2=$ positive and negative chondrocytes of approximately equal number; $1=$ more negative than positive chondrocytes; $0=$ all chondrocytes negative. Positive staining was recognised as a dark red colour associated with the cytoplasmic membrane or cytoplasm, or both. As chondrocytes were either strongly positive or exhibited no staining at all no grading of the positivity was made. Areas of sections that contained folding of the cartilage or exhibited non-specific background staining were not scored. Evaluation was carried out by two investigators and the scores given were agreed upon.

ETHICS

The study was approved by the regional ethics committee as part of a larger project involving articular cartilage and bone. Written information about the project was presented to patients scheduled for surgery and patient consent was obtained in these cases.

\section{STATISTICS}

The Friedman test was used to test the null hypothesis that there is no systematic topographical or zonal pattern of immunohistochemical scores in normal or osteoarthritic cartilage. Tests for systematic topographical pattern were performed for each zone for all normal femoral heads. Tests were not performed for osteoarthritic femoral heads because of insufficient numbers of available sections and, hence, data to compute. Tests for systematic zonal pattern were performed for the superficial zone versus the deep zone for each normal and osteoarthritic femoral head. The Mann-Whitney U test was used to test the

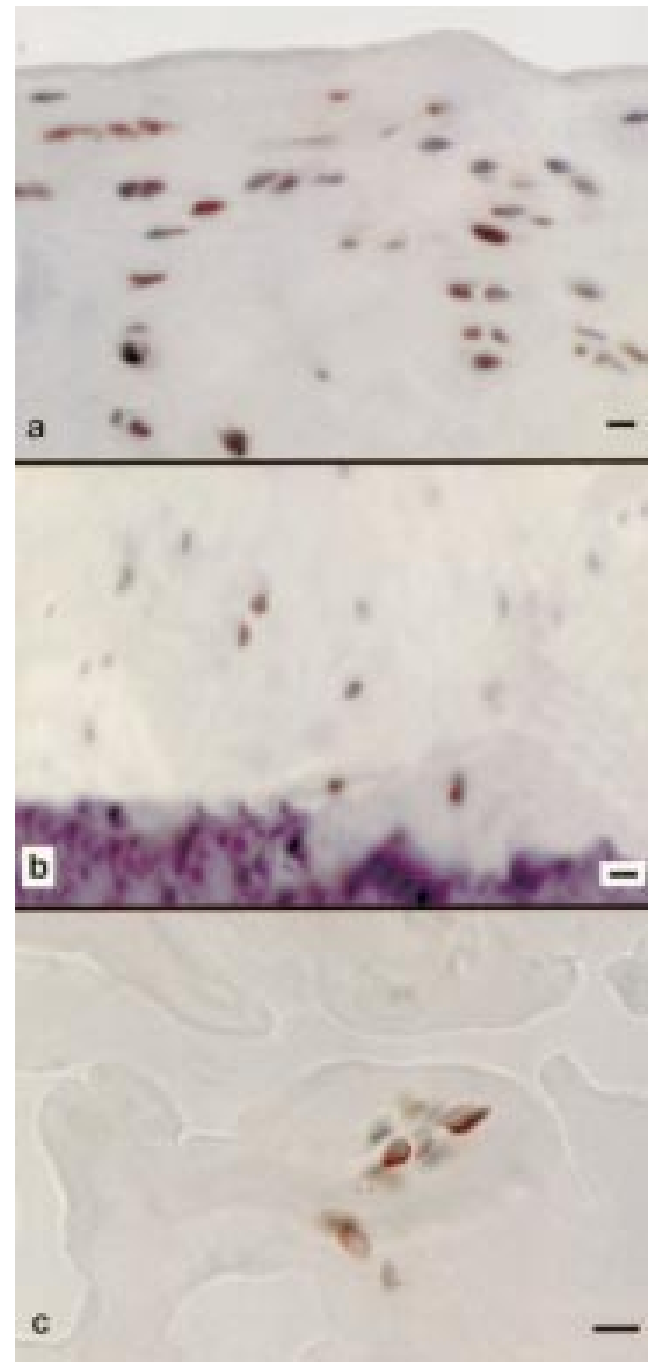

Figure 1 Articular cartilage stained for the a V integrin subunit. (a) The majority of chondrocytes are aV positive in the superficial zone of articular cartilage from a macroscopically normal femoral head. (b) Both aV positive and $a V$ negative chondrocytes in the deep zone of articular cartilage from a macroscopically normal femoral head. (c) $A$ cluster containing both $a V$ positive and $a V$ negative chondrocytes in non-intact articular cartilage from an osteoarthritic femoral head; the cartilage contains several clefts. Immunohistochemistry with an immunoperoxidase technique and haematoxylin counterstaining was used (bar marker: $25 \mu \mathrm{m}$ ).

null hypothesis that there is no systematic difference between immunohistochemical scores in normal and osteoarthritic cartilage. The limit of significance was chosen as 0.05 .

\section{Results}

NORMAL CARTILAGE

Forty eight to 70 sections of the possible 72 sections of cartilage from the six macroscopically normal femoral heads stained for the various $\alpha$ and $\beta$ subunits were available for evaluation (table 2). Sections were excluded for evaluation because the tidemark was not included or because of excessive non-specific background staining.

Table 2 presents a summary of results for all integrin subunits for articular cartilage from macroscopically normal femoral heads. There was no systematic pattern in the staining with respect to topographical site for any of the 
Table 4 Integrin subunits in intact and non-intact residual articular cartilage from osteoarthritic femoral heads

\begin{tabular}{lll}
\hline $\begin{array}{l}\text { Integrin subunit } \\
\text { (number of } \\
\text { sections stained) }\end{array}$ & Intact median score (range) & $\begin{array}{l}\text { Non-intact } \\
\text { median score } \\
\text { (range) }\end{array}$ \\
\hline$\alpha 1(41)$ & $2(0-3)$ & $2(1-3)$ \\
$\alpha 2(51)$ & $1(0-2)$ & $1(1-2)$ \\
$\alpha 3(47)$ & $0(-)$ & $0(-)$ \\
$\alpha 4(50)$ & $0(0-1)^{\star}$ & $0(0-1)^{\star}$ \\
$\alpha 5(48)$ & $2(0-3)$ & $2(1-3)$ \\
$\alpha 6(55)$ & $0(-)$ & $0(-)$ \\
$\alpha \mathrm{V}(35)$ & $3(2-3)$ superficial & \\
& $2(1-3)$ middle & $1(1-3)$ \\
$\beta 1(47)$ & $2(1-3)$ deep & $3(1-3)$ \\
$\beta 2(52)$ & $2(1-3)$ & $0(0-1)^{\star}$ \\
$\beta 3(54)$ & $0(0-1)^{\star}$ & $0(-)$ \\
$\beta 4(48)$ & $0(-)$ & $1(0-2)$ \\
$\alpha \mathrm{V} \beta 5(45)$ & $1(0-3)$ & $1(1-3)$ \\
$\beta 6(55)$ & $0(-)$ & $0(-)$ \\
& &
\end{tabular}

All topographical sites and zones of 4-6 subjects were scored together except for $\alpha \mathrm{V}$ subunit where the score is given for the superficial, middle, and deep zone individually. ${ }^{\star}=$ Cytoplasmic reaction in occasional chondrocytes. Scores: see table 2 .

integrin subunits ( $p>0.05$ ). Only the $\alpha \mathrm{V}$ subunit displayed a systematic pattern in the zonal staining (see below). Occasional $\beta 4$ positive chondrocytes were seen throughout the zones in approximately one third of the sections.

Table 3 presents the zonal distribution of $\alpha \mathrm{V}$ in articular cartilage from macroscopically normal femoral heads. All 12 topographical sites had a decreased number of $\alpha \mathrm{V}$ positive chondrocytes in the middle and deep zones compared with the superficial zone (fig 1). This zonal pattern was seen in $48 \%$ of sections and this represented a statistically significant difference for the superficial zone versus the deep zone for all femoral heads $(p \leqslant 0.05)$. In only one section did chondrocytes in the superficial zone stain to a lesser degree for $\alpha \mathrm{V}$ than cells in the middle and deep zones.

\section{OSTEOARTHRITIC CARTILAGE}

Thirty five to 55 sections of the possible 72 sections of cartilage from the six osteoarthritic femoral heads stained for the various $\alpha$ and $\beta$ subunits were available for evaluation (table 4). Sections were excluded for evaluation because correct orientation could not be determined, no articular cartilage was present in the section, or because of excessive non-specific background staining.

Table 4 presents a summary of results for all integrin subunits for intact and non-intact residual articular cartilage from osteoarthritic femoral heads. The median score for all topographical sites and zones combined is shown with ranges. There was no overt variation in the staining pattern with respect to topographical site for any of the integrin subunits. Only the $\alpha \mathrm{V}$ subunit displayed a systematic pattern in the staining with respect to zone (see below) (fig 1). In contrast with chondrocytes in normal articular cartilage, all sections except one contained occasionally $\alpha 2$ positive chondrocytes (fig 2). Occasional $\beta 4$ positive chondrocytes were seen throughout the zones in approximately half the sections. In approximately one third of sections occasional chondrocytes stained for $\alpha 4$. In approximately

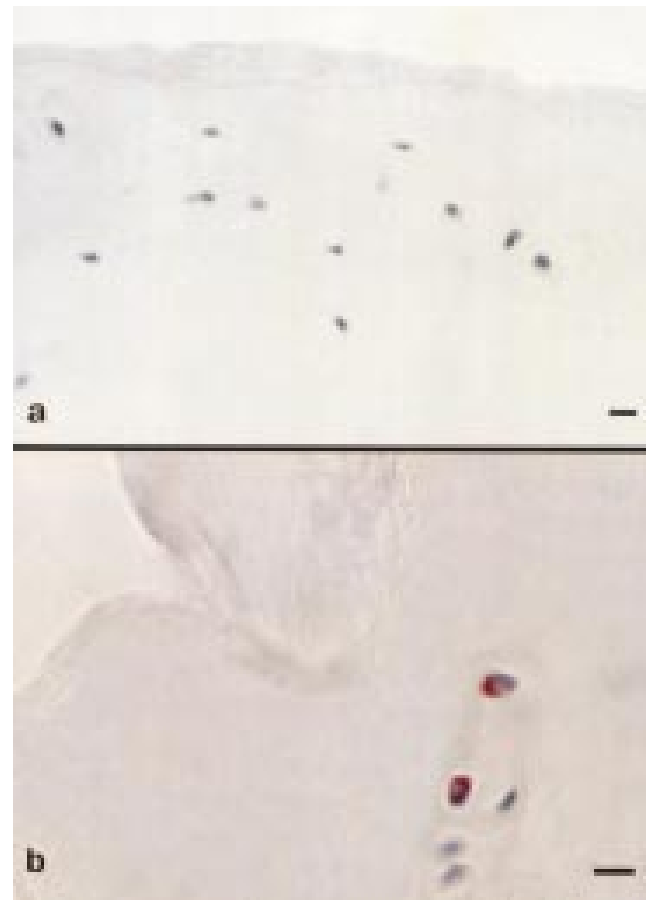

Figure 2 Articular cartilage stained for the a 2 integrin subunit. (a) a2 negative chondrocytes in the superficial zone of articular cartilage from a macroscopically normal femoral head. (b) A cluster containing both $\alpha 2$ positive and a2 negative chondrocytes in non-intact articular cartilage from an osteoarthritic femoral head. Immunohistochemistry with an immunoperoxidase technique and haematoxylin counterstaining was used (bar marker: $25 \mu \mathrm{m}$ ).

Table 5 Zonal distribution of a $V$ in intact and non-intact residual articular cartilage from osteoarthritic femoral heads

\begin{tabular}{|c|c|c|c|c|c|}
\hline Score & 0 & 1 & 2 & 3 & 4 \\
\hline \multicolumn{6}{|l|}{ Intact residual cartilage } \\
\hline Superficial zone & 0 & 0 & 9 & 21 & 0 \\
\hline Middle zone & 0 & 5 & 14 & 11 & 0 \\
\hline Deep zone & 0 & 12 & 8 & 10 & 0 \\
\hline \multicolumn{6}{|l|}{ Non-intact residual cartilage } \\
\hline Deep zone & 0 & 4 & 0 & 1 & 0 \\
\hline
\end{tabular}

Numbers for the three zones represent sections (30 for intact and five for non-intact residual articular cartilage from six patients) which have received a score. Scores: see table 2 .

$10 \%$ of sections occasional chondrocytes stained for $\beta 2$.

The differences in immunohistochemical staining scores between normal and osteoarthritic cartilage was not statistically significant for the various $\alpha$ and $\beta$ subunits ( $p>0.05$ ) except for the $\alpha 2$ and $\alpha 4$ subunits $(\mathrm{p} \leqslant 0.05)$.

Table 5 presents the zonal distribution of $\alpha \mathrm{V}$ in intact and non-intact residual articular cartilage from osteoarthritic femoral heads. The zonal pattern observed for normal cartilage was seen in $63 \%$ of sections and was statistically significant for the superficial zone compared with the deep zone for all femoral heads $(\mathrm{p} \leqslant 0.05)$. In the remaining sections, no zonal variation was observed.

Chondrocytes in clusters and in areas of excessive surface fibrillation showed a similar pattern of integrin subunit staining as isolated chondrocytes and chondrocytes in other areas of the same section.

The pre-treatment of sections with hyaluronidase, collagenase, or a combination thereof before the standard immunohistochemical 
staining did not reveal additional integrin subunit staining.

\section{Discussion}

This study confirmed the presence of integrin subunits $\alpha 1, \alpha 5, \alpha \mathrm{V}, \beta 1, \beta 4$, and $\beta 5$ and the absence of subunits $\alpha 2, \alpha 3, \alpha 4, \alpha 6, \beta 2, \beta 3$, and $\beta 6$ in normal human articular cartilage. ${ }^{91}$ The $\alpha 3$ subunit was absent in this study, but Salter et $a l^{11}$ found occasional $\alpha 3$ expression in all zones in femoral condylar and tibial plateaus cartilage. Woods et al could not detect the $\alpha 3$ subunit utilising immunohistochemistry, but these authors ${ }^{9}$ as well as Yonezawa et $a \beta^{4}$ confirmed the presence of this subunit by flow cytometric analysis. In this study, the $\beta 3$ subunit was also absent, confirming studies performed with immunohistochemistry ${ }^{911}$ and flow cytometric analysis. ${ }^{9}{ }^{34}$ However, Woods et $a l^{9}$ detected the heterodimer $\alpha \mathrm{V} \beta 3$ on chondrocytes within the most superficial $30 \mu \mathrm{m}$ of normal articular cartilage. Our detection of the $\beta 4$ subunit in occasional chondrocytes, but not the $\alpha 6$ subunit of the $\alpha 6 \beta 4$ heterodimer may represent a similar instance.

To our knowledge, this is the first study detailing the topographical and zonal distribution of integrins in osteoarthritic cartilage. In residual articular cartilage from osteoarthritic human femoral heads, chondrocytes expressed $\alpha 1, \alpha 5, \alpha \mathrm{V}, \beta 1, \beta 4$, and $\beta 5$. In addition, we found neo-expression of $\alpha 2, \alpha 4$, and $\beta 2$ in osteoarthritic cartilage. No staining was noted for $\alpha 3, \alpha 6, \beta 3$, and $\beta 6$. Jobanputra et al ${ }^{\beta 5}$ did not detect the subunits $\alpha 2$ and $\beta 4$, but did detect the $\alpha 3$ subunit in articular cartilage from the medial tibial plateaux of patients with osteoarthritis secondary to rheumatoid arthritis. We believe these differences in results reflect the differences in the selected material. In extracted chondrocytes from OA knee joints, the subunits $\alpha 1-6, \alpha \mathrm{V}$, and $\beta 1$ were all detected by flow cytometric analysis. ${ }^{36}$ The differences in results obtained in studies using immunohistochemistry or flow cytometric analysis may reflect either (1) masking of integrin subunits in cartilage sections eliminated in the process of chondrocyte extraction before flow cytometric analysis or (2) neoexpression of integrin subunits in response to the extraction process.

There was no topographical variation in the expression of subunits in either normal or osteoarthritic cartilage. The only subunit that displayed a zonal variation was $\alpha \mathrm{V}$. Minor variations in the expression of subunits with respect to topography or zones may have been missed because of the rather crude immunoscoring system utilised in this study. Interobserver and intraobserver reproducibilities of immunoscoring are rather low (unpublished data), however, the chosen immunoscoring system was found to be reproducible.

We have not attempted to correlate the integrin staining in residual cartilage from osteoarthritic femoral heads with a histopathological score obtainable through a grading system such as the histological-histochemical grading system for osteoarthritic articular cartilage $^{37}$ or modifications thereof. ${ }^{38} 39 \mathrm{Re}-$ cently, we have demonstrated low intraobserver and interobserver reproducibilities and questionable validity of the original histologicalhistochemical grading system for osteoarthritic articular cartilage. ${ }^{40}$

The OA articular cartilage selected for this study has been obtained from femoral heads graded III or IV according to the Collins/ McElligott system ${ }^{32}$ and, hence, represents end stage disease at least in some areas. The OA process in the non-intact residual cartilage of grade III and IV joints may thus be different from the $\mathrm{OA}$ process taking place in the non-intact residual cartilage of grade I and II joints. Unfortunately, without a reliable histopathological grading system it is difficult to obtain human articular cartilage representative of the different stages of the OA process. Animal studies may further elucidate the expression of $\alpha$ and $\beta$ subunits of the integrin superfamily during the different OA stages.

From the integrin subunits detected in this and previous ${ }^{911}$ studies, it is possible to deduce the presence of the following integrin heterodimers in normal and OA human articular cartilage: $\alpha 1 \beta 1, \alpha 2 \beta 1, \alpha 3 \beta 1, \alpha 4 \beta 1, \alpha 5 \beta 1, \alpha \mathrm{V} \beta 1$, $\alpha \mathrm{V} \beta 3, \alpha 6 \beta 4$, and $\alpha \mathrm{V} \beta 5$. The $\beta 2$ subunit may associate with the $\alpha \mathrm{L}, \alpha \mathrm{M}$, and $\alpha \mathrm{X}$ subunits. The heterodimers $\alpha 1 \beta 1$ and $\alpha 2 \beta 1$ are known receptors for collagens and laminin; $\alpha 3 \beta 1$ for collagens, fibronectin, and laminin; $\alpha 4 \beta 1$ for fibronectin, chondroitin sulphate, and VCAM (vascular cell adhesion molecule)-1; $\alpha 5 \beta 1$ for fibronectin; $\alpha \mathrm{V} \beta 1$ for vitronectin and fibronectin; $\alpha \mathrm{V} \beta 3$ for collagens, fibronectin, vitronectin, thrombospondin, and laminin; $\alpha 6 \beta 4$ for laminin; $\alpha \mathrm{V} \beta 5$ for vitronectin; $\alpha \mathrm{L} \beta 2$ for ICAM$1 / 2 / 3 ; \alpha M \beta 2$ for ICAM-1, fibrinogen, factor $\mathrm{X}$, and complement factor $\mathrm{iC} 3 \mathrm{~b}$; and $\alpha \mathrm{X} \beta 2$ for fibrinogen, denatured albumin and complement factor iC 3 b. ${ }^{13} 1441$

It is as yet unclear which extracellular matrix molecules human articular chondrocytes adhere to. Studies of the distribution of possible ligands such as fibronectin ${ }^{31}$ would suggest potential interaction. Such functional relations based upon codistribution is at best indicative and additional, possibly in vitro, studies are needed to unravel the precise role of integrin subunits in articular cartilage.

The significance of the neoexpression of $\alpha 2$, $\alpha 4$, and $\beta 2$ in residual cartilage from osteoarthritic femoral heads remains to be elucidated. The $\alpha 2$ subunit is strongly expressed in fetal cartilage $^{10}$ and the neoexpression in osteoarthritic cartilage of this subunit may represent an example of "fetal phenotype" expression in osteoarthritic cartilage potentially as a functional response to production of collagen type I and III. ${ }^{26}$

In conclusion, our study demonstrates the expression of $\alpha 1, \alpha 5, \alpha \mathrm{V}, \beta 1, \beta 4$, and $\beta 5$ in normal and osteoarthritic cartilage. In osteoarthritic cartilage, neoexpression of $\alpha 2, \alpha 4$, and $\beta 2$ is observed. There is no topographical variation in the expression of subunits in either normal or osteoarthritic cartilage. The only subunit that displays a zonal variation is $\alpha \mathrm{V}$; more chondrocytes express $\alpha \mathrm{V}$ in the superficial zone compared with the deep zone. 
We gratefully acknowledge Professor G Bendixen and Drs $M$ Brittberg and A Jordan for critical reviews of the manuscript and for helpful advice regarding statistical methods. Dr B Volck is acknowledged for the sampling of osteoarthritic cartilage. The skilful technical assistance of $\mathrm{V}$ Weibull and G Dahl is highly appreciated. The Michaelsen Foundation, the Danish Rheumatism Association, the Danish Medical Research Council, the Danish Biotechnology Program, the Arthritis and Rheumatism Council, and Direktør E Danielsen og Hustrus Fond are acknowledged for their financial support.

1 Lee GM, Johnstone B, Jacobson K, Caterson B. The dynamic structure of the pericellular matrix on living cells. J Cell Biol 1993;123:1899-907.

2 Benya PD, Shaffer JD. Dedifferentiated chondrocytes reexpress the differentiated collagen phenotype when cultured in agarose gels. Cell 1982;30:215-24.

3 Huang D. Extracellular Matrix-cell interactions and chondrogenesis. Clin Orthop 1976;123:169-76.

4 Arner EC, Tortorella MD. Signal transduction through chondrocyte integrin receptors induces matrix metalloproteinase synthesis and synergizes with interleukin-1. Arthritis Rheum 1995;38:1304-14.

5 Salter DM, Godolphin JL, Gourlay MS, Lawson MF, Salter DM, Godolphin JL, Gourlay MS, Lawson MF,
Hughes DE, Dunne E. Analysis of human articular Hughes DE, Dunne E. Analysis of human articular
chondrocyte CD44 isoform expression and function in chondrocyte CD44 isoform expression and func

6 Ostergaard K, Salter DM, Andersen CB, Petersen J, Bendtzen K. CD44 expression is up-regulated in the deep zone of osteoarthritic cartilage from human femoral heads. Histopathology 1997;31:451-9.

7 Jobanputra P, Corrigall V, Kingsley G, Panayi G. Cellular responses to human chondrocytes: Absence of allogeneic responses in the presence of HLA-DR and ICAM-1. Clin Exp Immunol 1992;90:336-44.

8 Davies ME, Dingle JT, Pigott R, Power C, Sharma H. Expression of intercellular adhesion molecule 1 (ICAM-1) on human articular cartilage chondrocytes. Connect Tissue Res 1991;26:207-16.

9 Woods VL, Jr., Schreck PJ, Gesink DS, Pacheco HO, Amiel $\mathrm{D}$, Akeson WH, et al. Integrin expression by human articular chondrocytes. Arthritis Rheum 1994;37:537-44.

10 Salter DM, Godolphin JL, Gourlay MS. Chondrocyte heterogeneity: immunohistologically defined variation of heterogeneity: immunohistologically defined variation of integrin expression at different sites in h

11 Salter DM, Hughes DE, Simpson R, Gardner DL. Integrin expression by human articular chondrocytes. Br J Rheumatol 1992;31:231-4

12 Dürr J, Goodman S, Potocnik A, von der Mark H, von de Mark K. Localization of beta 1-integrins in human cartilage and their role in chondrocyte adhesion to collagen and fibronectin. Exp Cell Res 1993;207:235-44.

13 Hynes RO. Integrins: Versatility, modulation, and signaling in cell adhesion. Cell 1992;69:11-25.

14 Loeser RF. Integrin-mediated attachment of articular chondrocytes to extracellular matrix proteins. Arthritis Rheum 1993;36:1103-10.

15 Armstrong CG, Gardner DL. Thickness and distribution of human femoral head articular cartilage. Changes with age. human femoral head articular car

16 Bayliss MT, Davidson C, Woodhouse SM, Osborne DJ. Chondroitin sulphation in human joint tissues varies with age, zone and topography. Acta Orthop Scand Suppl 1995; age, zone and

17 Mitrovic D, Quintero M, Stankovic A, Ryckewaert A. Cell density of adult human femoral condylar articular cartilage. Joints with normal and fibrillated surfaces. Lab Invest 1983;49:309-16.

18 Bayliss MT, Venn M, Maroudas A, Ali SY. Structure of proteoglycans from different layers of human articula cartilage. Biochem J 1983;209:387-400.

19 Hamerman D. The biology of osteoarthritis. N Engl J Med 1989;320:1322-30.

20 Poole AR. Changes in the collagen and proteoglycan of articular cartilage in arthritis. Reumatology 1986;10:31671.
21 Maroudas A, Venn M. Chemical composition and swelling of normal and osteoarthrotic femoral head cartilage. II. Swelling. Ann Rheum Dis 1977;36:399-406.

22 Venn M, Maroudas A. Chemical composition and swelling of normal and osteoarthrotic femoral head cartilage. I. Chemical composition. Ann Rheum Dis 1977;36:121-9.

23 von der Mark K, Kirsch T, Nerlich A, Kuss A, Weseloh G, Gluckert $\mathrm{K}$, et al. Type X collagen synthesis in human osteoarthritic cartilage. Indication of chondrocyte hypertrophy. Arthritis Rheum 1992;35:806-11.

24 Gay S, Muller PK, Lemmen C, Remberger K, Matzen K, Kuhn K. Immunohistological study on collagen in
cartilage-bone metamorphosis and degenerative osteoarthrosis. Klin Wochenschr 1976;54:969-76.

25 Adam M, Deyl Z. Altered expression of collagen phenotype in osteoarthrosis. Clin Chim Acta 1983;133:25-32.

26 Aigner T, Dietz U, Stoss H, von der Mark K. Differential expression of collagen types I, II, III, and X in human osteophytes. Lab Invest 1995;73:236-43.

27 Ronziere MC, Ricard Blum S, Tiollier J, Hartmann DJ, Garrone R, Herbage D. Comparative analysis of collagens solubilized from human foetal, and normal and osteoarthritic adult articular cartilage, with emphasis on type VI collagen. Biochim Biophys Acta 1990;1038:222-30.

28 Nemeth-Csoka M, Meszaros T. Minor collagens in arthrotic human cartilage. Change in content of 1 alpha, 2 alpha, 3 alpha and M-collagen with age and in osteoarthrosis. Acta Orthop Scand 1983;54:613-9.

29 Walker GD, Fischer M, Gannon J, Thompson RC Jr, Oegema TR. Expression of type-X collagen in osteoarthritis. J Orthop Res 1995;13:4-12.

30 Miller DR, Mankin HJ, Shoji H, D'Ambrosia RD. Identification of fibronectin in preparaons of osteoarthritic human cartilage. Connect Tissue Res 1984;12:267-75.

31 Jones KL, Brown M, Ali SY, Brown RA. An immunohistochemical study of fibronectin in human osteoarthritic and disease free articular cartilage. Ann Rheum Dis 1987;46: $809-15$

32 Collins DH, McElligott TF. Sulphate $\left({ }^{35} \mathrm{SO}_{4}\right)$ uptake by chondrocytes in relation to histological changes in osteoarthritic human articular cartilage. Ann Rheum Dis 1960;19: 318-30.

33 Hughes DE, Salter DM, Dedhar S, Simpson R. Integrin expression in human bone. J Bone Miner Res 1993;8:52733.

34 Yonezawa I, Kato K, Yagita H, Yamauchi Y, Okumura K. VLA-5-mediated interaction with fibronectin induces cytokine production by human chondrocytes. Biochem Biophys Res Commun 1996;219:261-5.

35 Jobanputra P, Lin H, Jenkins K, Bavington C, Brennan FR, Nuki G, et al. Modulation of human chondrocyte integrins by inflammatory synovial fluid. Arthritis Rheum 1996;39: 1430-2.

36 Lapadula G, Iannone F, Zuccaro C, Grattagliano V, Covelli M, Patella V, et al. Integrin expression on chondrocytes: correlations with the degree of cartilage damage in human correlations with the degree of cartilage damage in hum
osteoarthritis. Clin Exp Rheumatol 1997;15:247-54.

37 Mankin HJ, Dorfman H, Lippiello L, Zarins A. Biochemical and metabolic abnormalities in articular cartilage from osteo-arthritic human hips. II. Correlation of morphology with biochemical and metabolic data. J Bone Joint Surg Am 1971;53:523-37.

$38 \mathrm{Kim}$ HK, Moran ME, Salter RB. The potential for regeneration of articular cartilage in defects created by chondral shaving and subchondral abrasion. An experimental investigation in rabbits. J Bone Joint Surg Am 1991; 73:1301-15.

39 Bulstra SK, Buurman WA, Walenkamp GH, van der Linden AJ. Metabolic characteristics of in vitro cultured human chondrocytes in relation to the histopathologic grade of osteoarthritis. Clin Orthop 1989;242:294-302.

40 Ostergaard K, Petersen J, Andersen CB, Bendtzen K, Salter DM. Histologic/histochemical grading system for osteoarthritic articular cartilage: reproducibility and validity. Arthritis Rheum 1997;40:1766-71.

41 Ruoslahti E. Integrins. J Clin Invest 1991;87:1-5. 